\title{
Capital social e Sistema Único de Assistência Social (SUAS) no Rio Grande do Sul
}

\author{
Social capital and Unified Social Assistance System (SUAS) in \\ Rio Grande do Sul State
Capital social y el Sistema Único de Asistencia Social (SUAS) en Rio Grande do Sul

\author{
Douglas Marques ${ }^{1}$ \\ Everton Rodrigo Santos ${ }^{2}$ \\ Cristina Ennes da Silva ${ }^{2}$
}

Recebido em 20/01/2019, revisado e aprovado em 02/10/2019, aceito em 31/10/2019 DOI: http://dx.doi.org/10.20435/inter.v21i1.2358

\begin{abstract}
Resumo: O presente artigo baseia-se na teoria de Putnam, buscando verificar o impacto do capital social no aspecto da participação no Sistema Único de Assistência Social (SUAS) no Rio Grande do Sul, em especial nas cidades de Caxias do Sul e de Novo Hamburgo. Pretendemos demonstrar, comparativamente, a relação entre o acúmulo de capital social e a qualidade da participação nas políticas públicas. Utilizamo-nos de pesquisa de opinião pública, tipo Survey, por meio de duas baterias de testes nas cidades em tela, totalizando 382 questionários aplicados diretamente aos usuários do SUAS. Estatisticamente, foi possível afirmar nossa hipótese, qual seja, de que existe relação positiva entre capital social e a qualidade da participação nas políticas públicas, assim, na medida em que cresce o estoque de capital social, cresce a participação no âmbito das políticas públicas, em especial no SUAS.
\end{abstract}

Palavras-chave: capital social; políticas públicas; SUAS; participação; Rio Grande do Sul.

Abstract: The present study is based on Putnam's theory of social capital and its impact related to the participation aspect in the Unified Social Assistance System (SUAS) in Rio Grande do Sul State, especially in the cities of Caxias do Sul and Novo Hamburgo. In this way, we intend to demonstrate comparatively the relationship between the accumulation of social capital and the quality of participation in the SUAS. Regarding the methodological delimitation, we used a Survey research and 382 questionnaires were directly applied to SUAS users. In this sense, it was possible to affirm our hypothesis, that there is a positive relationship between social capital and the quality of participation in the public policy, thus, us to affirm that in so far as the stock of social capital grows, also occurs the growth of participation in the SUAS.

Keywords: social capital; public policy; SUAS; participation; Rio Grande do Sul.

Resumen: El presente artículo se basa en la teoría de capital social de Putnam y su impacto en el aspecto de la participación en el Sistema Único de Asistencia Social (Suas) en Rio Grande do Sul, especialmente en las ciudades de Caxias do Sul y de Novo Hamburgo. La pretensión es relacionar la asociación existente en capital social y la calidad de la participación en las políticas públicas. Se utilizó de encuesta de opinión pública, tipo Survey, por medio de dos baterías de pruebas en las ciudades en pantalla, totalizando 382 cuestionarios aplicados directamente a los usuarios del Sistema Único de Asistencia Social (SUAS). En este sentido, estadísticamente fue posible afirmar nuestra hipótesis, cuál es, que existe relación positiva entre capital social y la calidad de la participación en las políticas públicas, así, en la medida en que crece la cantidad del capital social, crece la participación en el ámbito de las políticas públicas.

Palabras clave: capital social; políticas públicas; SUAS; participación; Río Grande do Sul.

\section{INTRODUÇÃO}

O recente relatório da revista The Economist aponta para o declínio dos governos democráticos no mundo nos últimos anos, considerando que apenas $5 \%$ da população vive em uma

\footnotetext{
${ }^{1}$ Universidade Estadual de Maringá (UEM), Maringá, Paraná, Brasil.

2 Universidade Feevale, Novo Hamburgo, Rio Grande do Sul, Brasil.
} 
"democracia plena". Acompanhando esta tendência, o Brasil foi classificado no nível mais baixo, de "democracias com falhas" ${ }^{3}$. Num primeiro momento, olhando-se da perspectiva institucional (NORTH, 2007), o esforço dependeria exclusivamente das leis, das instituições e dos gestores públicos. Todavia, numa perspectiva culturalista (PUTNAM, 2000), seríamos levados a observar que os aspectos socioculturais de uma determinada região têm influência direta na qualidade do desempenho e na participação nas políticas públicas. Assim, particularmente nos interessa, por razões socioculturais, estudar as cidades de Novo Hamburgo e Caxias do Sul.

Muito embora nas últimas décadas a sociedade brasileira tenha experimentado um crescimento significativo da descentralização de políticas públicas, persistem ainda os dilemas para que essas políticas estejam de fato voltadas para o aprofundamento da democracia e da participação da sociedade. Participação entendida, no âmbito do SUAS, por meio de práticas e mecanismos que favoreçam o processo de planejamento e a execução da Política Nacional de Assistência Social de modo democrático e participativo (BRASIL, 2014).

Nesta direção, de forma original no Brasil, este trabalho busca, à luz da teoria do capital social, analisar comparativamente a qualidade da participação dos usuários do Sistema único de Assistência Social (SUAS) nas cidades de Caxias do Sul e Novo Hamburgo, no Rio Grande do Sul. Nessa perspectiva, a nossa hipótese de trabalho sugere, a partir da relação definida por Putnam (2000), que o acúmulo de capital social existente numa determinada região, ou seja, suas relações de confiança interpessoal, de participação política e a cultura cívica, está positivamente correlacionado com o fortalecimento e a ampliação da participação nas políticas públicas. A metodologia, utiliza resultados de pesquisa quantitativa, tipo Survey, realizada em 2016, formada por duas baterias de testes, uma na cidade de Caxias do Sul, constituída de 211 questionários, outra na cidade de Novo Hamburgo, constituída de 171 questionários, totalizando $382^{4}$, com erro amostral de $5 \%$ e coeficiente de confiança de $95 \%$. Os questionários foram aplicados diretamente aos usuários do SUAS, maiores de 18 anos e referenciados aos aparelhos públicos Centro de Referência de Assistência Social (CRAS) e Centro de Referência Especializado de Assistência Social (CREAS), levando em conta as normas aplicáveis a pesquisas em Ciências Sociais e Humanas, segundo a Res. 510/16, do Ministério da Saúde.

Assim, num primeiro momento, buscaremos apresentar nossa compreensão sobre as políticas públicas, sugerindo a relevância da cultura política como elemento de sustentação da democracia, no contexto brasileiro; em seguida, lançaremos o debate sobre o capital social como vetor de desenvolvimento, em especial, para o fortalecimento da participação nas políticas públicas. Por fim, demonstraremos, a partir de uma perspectiva socioculturalista, a associação positiva entre capital social e participação no SUAS, nas cidades em tela.

\section{A CONTRIBUIÇÃO DO CAPITAL SOCIAL PARA AS POLÍTICAS PÚBLICAS}

Ao analisarmos o contexto brasileiro, os estudos sobre as políticas públicas têm início no século XX, muito embora nos anos 1980 haja uma retomada das análises sobre políticas de gestão dos governos, de políticas públicas e de políticas em geral. Segundo Miceli (1999), a tradição de

\footnotetext{
${ }^{3}$ Em 2014, a pontuação do Brasil era de 7.38 para um máximo de 10. Em 2017, a pontuação caiu para 6.86, ficando perto de um "regime híbrido". Disponível em: http://economistbrazil.com.br/pt_br/Noticias.aspx?id=2. Acesso em: 13 mar. 2018.

${ }^{4}$ Segundo Barbetta, 2010.
} 
análise das políticas públicas segue a escola anglo-saxônica, mais especificamente a norte-americana, pelos pressupostos do 'antiestatismo liberal' e pelos estudos da cultura política, ensejando os estudos sobre "o Estado em ação". Desta forma, os estudos sobre as políticas públicas têm se dividido em três campos predominantes: 1) Regime político, instituições políticas ou Estado; 2) Políticas setoriais e seus problemas internos e 3) Avaliação de políticas públicas (MICELI, 1999).

Sobre os tipos de políticas públicas, a literatura especializada tem cotejado diferentes abordagens, como a atenção às dimensões técnica e política de Bozeman e Pandey (2004); a distinção entre as políticas reais, simbólicas e pseudopolíticas de Gustafsson (1983); as políticas do tipo clientelista, de grupo de interesses, empreendedoras e majoritárias de Wilson (1983); as abordagens de políticas regulatórias, distributivas, constitutivas e redistributivas de Lowi (1964); e a operacionalização de políticas públicas pelos modelos: principal-agente, rede de políticas públicas, elitista e pluralista, de Secchi (2014).

Neste sentido, as políticas públicas têm se tornado um campo do conhecimento em grande expansão na atualidade, servindo, contudo, para múltiplas interpretações e tornando difuso o seu conceito. Nesta direção, na perspectiva de Secchi (2014, p. 2), "política pública é uma diretriz elaborada para enfrentar um problema público, constituindo-se de uma intencionalidade pública e a resposta a um problema público". Em outro ponto de vista, Lahera (2012) sugere que as políticas públicas indicam a ação de uma autoridade pública ao colocar em marcha uma política ou programa. Na compreensão de Pase e Santos (2011), as políticas públicas são um conjunto de ações do Estado, postas em marcha mediante programas e projetos para efetivar concepções acerca da saúde, da educação, da assistência social, da segurança, da economia, da infraestrutura, entre outras. Para os limites deste trabalho, respaldados por Souza (2006, p. 26), entendemos política pública como

O campo do conhecimento que busca, ao mesmo tempo, "colocar o governo em ação" e/ou analisar essa ação (variável independente) e, quando necessário, propor mudanças no rumo ou curso dessas ações (variável dependente). Em outras palavras, o processo de formulação de política pública é aquele através do qual os governos traduzem seus propósitos em programas e ações, que produzirão resultados ou as mudanças desejadas no mundo real.

Observa-se uma tensão embrionária na formulação das políticas públicas, expressando-se na bifurcação: a implantação das políticas públicas tem sido outorgada pelo Estado de "cima para baixo", enquanto a sua implementação dependerá, grosso modo, da iniciativa da sociedade, influenciando de "baixo para cima". Dentro do quadro das políticas públicas que compõe a Seguridade Social no Brasil (BRASIL, 1988), nossa abordagem volta-se para a Política Nacional de Assistência Social (PNAS), que vem sendo operacionalizada por meio do SUAS (BRASIL, 2005, p. 33), no qual "as ações são organizadas em sistema descentralizado e participativo, constituído pelas entidades e organizações de assistência social, articulando meios, esforços e recursos, e por um conjunto de instâncias deliberativas", composto por dois níveis de proteção social: básica e especial. Desta forma, a expansão e a descentralização do SUAS vêm sendo acompanhadas dos esforços e desafios da gestão pública e da sociedade para implementá-lo da melhor maneira possível em contextos e regiões diferentes no país, particularmente no Rio Grande do Sul.

Considerando a inclinação da experiência brasileira para estudos de políticas públicas voltados para a ação do Estado, numa perspectiva institucionalista, coloca-se a problemática: as políticas públicas dependeriam exclusivamente das instituições políticas para promover um bom desempenho institucional? Ou as políticas públicas teriam de contar com elementos da cultura 
política e com recursos sociais como vetores para o seu bom desempenho? Considerando a crise das instituições políticas e o cenário de tensões entre Estado e a sociedade, a bibliografia tem se bifurcado entre duas grandes tradições de estudos, o institucionalismo (NORTH, 2007) e o culturalismo (PUTNAM, 2000), para explicar o desempenho institucional dos governos.

Neste sentido, mesmo considerando a realização de eleições limpas, a eficiência governamental e o consenso entre algumas das principais tarefas da democracia, a sua estabilidade necessita de uma infraestrutura social adequada (FERRAZ, 2016). O aspecto da infraestrutura social envolve a atenção também de Baquero (2013), na medida em que ele utiliza o conceito de "democracia inercial" para sugerir que, no Brasil, por meio das teorias de escolhas racional e institucional, afirmava-se que as instituições formais, aos poucos, seriam capazes de solucionar (automaticamente) os problemas sociais e políticos, portanto as instituições atuariam como variável independente. Contudo essa perspectiva não tem se comprovado, pois, quando crises econômicas se instauram, elas produzem crises de credibilidade nas instituições, colocando em xeque a própria legitimidade de um governo democraticamente eleito (BAQUERO, GONZALEZ 2016). O fato fica evidente na atualidade, conforme última pesquisa de opinião pública sobre a avaliação do Governo Federal, em que 30\% da população o considera um ótimo governo, 32\% regular e 38\% ruim/péssimo ${ }^{5}$ (DATAFOLHA, 2019).

Diante deste panorama, há uma volta aos estudos do capital social e da cultura política como elementos imprescindíveis para sustentação das instituições democráticas (PUTNAM, 2000; ARROW, 1974; DOWBOR, 2003; GIDDENS, 2007; TABELLINI, 2005; BAQUERO, 2007; SANTOS, 2013, entre outros). Desta forma, o capital social, como um conceito polissêmico, abrangendo os aspectos sociológico, econômico, político e cultural, pode ser uma proposta estimulante. Um dos primeiros usos do termo "capital social" na literatura acadêmica foi feito por Hanifan (1916), relacionado a uma abordagem em um centro comunitário rural no Estado da Virgínia Ocidental, nos Estados Unidos. Diante de várias interpretações do conceito de capital social, temos a perspectiva histórica e econômica de Wittman, Ramos (2004, p. 42); o ponto de vista de Szreter (1999), em que as relações de confiança e a qualidade das relações entre grupos sociais são as bases constitutivas de capital social; e, para Fukuyama (1996, p. 41), "a compreensão da vida econômica está intrinsecamente ligado à vida social, onde o capital social decorre da prevalência de confiança numa sociedade".

Entre outros importantes autores das ciências sociais que vêm contribuindo para o debate conceitual sobre o capital social, citamos Bourdieu (1980) e Coleman (1988). Para o primeiro autor, trata-se de um "conjunto de recursos atuais ou potenciais que são colocados à posse de uma rede durável de relações mais ou menos institucionalizadas pelo convívio e pelo reconhecimento; ou, em outras palavras, ao pertencimento de um grupo, como um conjunto de agentes que não são apenas dotados de propriedades comuns, mas são também unidos por laços permanentes e úteis" (BOURDIEU, 1980, p. 80). Para o segundo autor, "o capital social é definido por sua função. Ele não é uma entidade individual, mas uma variedade de diferentes entidades com dois elementos comuns: todas elas consistem em algum aspecto das estruturas sociais e elas facilitam certas ações dos atores - sejam pessoas ou atores corporativos - dentro da estrutura" (COLEMAN, 1988, p. 98).

\footnotetext{
${ }^{5}$ Pesquisa Survey realizada com 2.948 pessoas nos dias 5 e 6 do mês de dezembro de 2019, erro amostral de $2 \%$ e confiança de $95 \%$.
} 
Contudo nosso entendimento sobre capital social recai sobre a teoria de Putnam (2000), em que as instituições são importantes marcos político-legais que tencionam mudanças na sociedade, mas essas são moldadas pelo contexto sociocultural no qual estão inseridas. Nessa perspectiva de análise, investe-se nos aspectos socioculturais como dispositivos de propensão e acumulação de capital social, influenciando positivamente no desempenho institucional, e não o contrário. Desta forma, a confiança interpessoal de uma sociedade, o seu engajamento cívico e a sua participação política estão na base do sucesso de instituições democráticas estáveis e eficazes. Nesse sentido, Putnam (2000), ao pesquisar a realidade italiana por mais de 20 anos, tem como pressupostos teóricos compreender quais são as condições necessárias para a criação instituições fortes, eficazes e responsáveis. Assumindo um viés culturalista, o autor vai atrelar características do contexto sociocultural para explicar porque o norte da Itália teve melhor desempenho institucional e capacidade de governança em relação ao sul da Itália. Assim, capital social, para Putnam (2000, p. 137), diz respeito ao

Conjunto de recursos sociais possuído por um grupo, através de redes de trabalho com as quais se constitui uma comunidade cívica, sentimentos de solidariedade e igualdade com os demais membros da comunidade, normas de cooperação, reciprocidade, confiança e atitudes positivas, reveladas através da confiança no outro, no governo e no funcionamento das instituições.

Com este entendimento, Putnam (2000) conclui a existência de uma significativa associação entre engajamento cívico, redes de confiança interpessoal e o desempenho das instituições políticas. Conforme já apontou Inglehart (2001, p. 88), o debate pode ser ampliado, pois "ciertas variables culturales, económicas y políticas guardan una relación estrecha". Nesta direção, recentes pesquisas indicam que o capital social está associado com o fortalecimento e o empoderamento da democracia (BAQUERO, 2013), com a ampliação da confiança nas instituições do estado (GIDDENS, 2007) e para o melhor desempenho das políticas públicas (BAQUERO, 2013; SANTOS, 2013). Neste último aspecto, Santos (2013), ao estudar a relação entre democracia e capital social no Rio Grande do Sul, sugere que futuras pesquisas oportunamente poderiam explorar bem mais a relação entre capital social e participação societal.

Neste sentido, o tema de participação está entre os maiores desafios postos às políticas públicas pós-constituintes, bem como é uma das temáticas que mais se têm produzido no Brasil, sobretudo nas últimas duas décadas, por diferentes abordagens (ROUSSEAU, 1968; OLSON, 1999; STASSEN, 1999; MILL, 2001; TOCQUEVILLE, 1998; BOBBIO et al., 1998; GOHN, 2011, entre outros).

No âmbito do SUAS, por meio da Lei Orgânica de Assistência Social (LOAS), n. 8.742/1993, no seu art. 5으, busca-se assegurar a participação da população por meio de organizações representativas na formulação das políticas e no controle das ações em todos os níveis federativos (municipal, estadual e federal). Neste sentido, a participação é compreendida como uma estratégia presente na gestão do SUAS, por meio da adoção de práticas e mecanismos que favoreçam o planejamento e a execução de modo democrático e participativo, em especial, por meio das conferências nos âmbitos municipal, estadual e nacional (BRASIL, 2005).

Desta forma, na sequência deste artigo, analisaremos a participação no SUAS, partindo de um nível inexpressivo, indo até um nível mais sofisticado (BORDENAVE, 1983); assim: em nível de informação, a população é apenas informada das decisões já tomada pelos governantes; em nível de consulta, a população é consultada em certas ocasiões, embora a decisão final pertença aos governantes; em nível de elaboração, a população é convidada a expressar críticas ou sugestões, 
sendo os governantes obrigados a justificarem as suas escolhas; por fim, em nível de delegação, a população escolhe os objetivos e os meios para resolver os problemas.

\section{A PARTICIPAÇÃO NO SUAS DO RIO GRANDE DO SUL: CAPITAL SOCIAL É A RESPOSTA?}

No Brasil, em especial na formação sociocultural do Rio Grande do Sul (PESAVENTO, 1980; GERTZ, 2011), pesquisas vêm replicando o arcabouço teórico de Putnam (2000), buscando explicar significativos contrastes regionais entre o "Norte gaúcho", com pouca escravidão e a presença do minifúndio; e o "Sul gaúcho", com a presença marcante do latifúndio e do trabalho escravo (BANDERA, 2003; SANTOS, 2013, BAQUERO; PRÁ, 2007, PASE, 2016). Dessa forma, pesquisas têm sugerido um acúmulo maior de capital social no Norte, em especial na cidade de Caxias do Sul (BANDERA, 2003; BASTOS, 2008), muito embora constata-se também a presença positiva do capital social no município de Novo Hamburgo (SANTOS, 2013; 2016), indicando, assim, a constituição de uma comunidade cívica e que poderia haver um melhor funcionamento das instituições e das políticas públicas. Este acúmulo de capital social, para os limites deste trabalho, não guarda uma relação direta com as questões culturais e/ou étnicas nessas cidades, como resultado daquilo que tem sido chamado pela literatura do "mito da italianidade", em Caxias do Sul (SANTOS; ZANINI, 2009; HERÉDIA, 2005; CARAGNATO, 2010), e do "espírito obreiro do alemão", em Novo Hamburgo (SCHÜTZ, 1976), numa perspectiva antropológica. Contudo nosso trabalho está voltado para o tipo de organização social e para as práticas políticas dos agentes sociais erguidas ao longo da formação destas cidades, voltando nosso olhar para os aspectos de participação em grupos e associações, a confiança nas pessoas e nas instituições, o envolvimento cívico, entre outros, assim, numa perspectiva sociológica.

Portanto, em relação à cidade de Novo Hamburgo, esta foi marcada pela colonização alemã, no início do século XIX, constituindo-se em um distrito da cidade de São Leopoldo, sendo emancipada em 5 de abril de 1927. Está localizada a 40 quilômetros da capital do Estado, Porto Alegre, integrando a região do Vale do Rio dos Sinos. A cidade é reconhecida como a Capital Nacional do Calçado, com Produto Interno Bruto (PIB) de R\$ 8.122.335,91 (mil) e Índice de Desenvolvimento Humano Municipal (IDHM) de 0,747, possuindo uma população de cerca de 238.940 mil habitantes (IBGE, 2012). Já a cidade de Caxias do Sul é marcada predominantemente pela colonização italiana, no início do século XIX, tendo início no lugarejo de Colônia de Caxias, sendo elevada à cidade em 1으 de julho de 1910. Está localizada a 128 quilômetros da capital do Estado do Rio Grande do Sul, integrando a região da Serra. A cidade integra, junto a outros municípios, uma região reconhecida pela produção de vinho e derivados, com PIB de R\$ 20.637.191,76 e IDHM de 0,782, tendo uma população de 435.564 habitantes (IBGE, 2012).

Neste sentido, somados aos aspectos da formação sociocultural e ao acúmulo do capital social, para a escolha dos municípios, foram levados em consideração também critérios institucionais, tais como: ambos os municípios são considerados de grande porte ${ }^{6}$; ambos têm o nível de gestão plena do SUAS ${ }^{7}$ e ambos dispõem da mesma quantidade de aparelhos públicos, sendo 6 (seis) CRAS e 2 (dois) CREAS. Desta forma, coloca-se a problemática: considerando, na sua

\footnotetext{
${ }^{6}$ Entende-se por municípios de grande porte aqueles cuja população é de 101.000 habitantes até 900.000 habitantes, com cerca de 25.000 a 250.000 famílias (BRASIL, 2005).

${ }^{7}$ Gestão total das ações de assistência social, com foco em organizar a proteção social básica e especial (BRASIL, 2005).
} 
maioria, as semelhanças institucionais dos municípios, seria o capital social o vetor relacionado com a qualidade da participação no SUAS?

Nesta direção, consideramos que é possível medir o seu estoque a partir de informações sobre o envolvimento cívico, além de participação política e da confiança interpessoal da população (RIBEIRO; FERNANDES; RIBEIRO, 2012; INGLEHART, 2001). Neste sentido, foram estabelecidas algumas afirmações com relação a hábitos e costumes da população, em que, para cada afirmação positiva, foi atribuído valor 2; para cada negativa, valor 0; e, para cada possibilidade remota ("às vezes", "um pouco"), o valor 1. Desta forma, o Índice de Capital Social (ICS) é formado pela agregação das duas primeiras respostas (indicando as respostas positivas) e pela agregação da última resposta (indicando a resposta negativa). Assim sendo, para aferirmos o acúmulo de capital social nas cidades em tela, inspirados em Khan e Silva (2012), estabelecemos o seguinte critério: baixo nível de capital social $-0<I C S \leq 0,5$; médio nível de capital social $-0,5<$ ICS $\leq 0,8$; alto nível de capital social $-0,8<\mathrm{ICS} \leq 1$, conforme a Tabela 1 abaixo.

Tabela 1 - Índice de Capital Social das cidades de Caxias do Sul e Novo Hamburgo

\begin{tabular}{|c|c|c|c|}
\hline \multirow{2}{*}{\multicolumn{2}{|c|}{ Indicador }} & Caxias do Sul & Novo Hamburgo \\
\hline & & Valor & Valor \\
\hline \multicolumn{2}{|l|}{ Confiança nas pessoas } & 0,313 & 0,246 \\
\hline \multicolumn{2}{|l|}{ Confiança nas instituições } & 0,380 & 0,388 \\
\hline \multicolumn{2}{|l|}{ Participação em grupos e associações } & 0,190 & 0,165 \\
\hline \multicolumn{2}{|l|}{ Resolver problemas com outras pessoas } & 0,379 & 0,327 \\
\hline \multicolumn{2}{|l|}{ Conhecimento sobre grupos no bairro } & 0,335 & 0,286 \\
\hline \multicolumn{2}{|l|}{ Envolvimento em grupo ou organização } & 0,261 & 0,240 \\
\hline \multicolumn{2}{|l|}{ Confiança nos vizinhos } & 0,294 & 0,280 \\
\hline \multicolumn{2}{|l|}{ Redes de amizade } & 0,250 & 0,250 \\
\hline \multirow{2}{*}{ Engajamento cívico } & & 0,896 & 0,842 \\
\hline & Total & 0,366 & 0,336 \\
\hline
\end{tabular}

Total: Caxias do Sul N = 211 - Novo Hamburgo N = 171 .

Fonte: Próprios autores.

De uma maneira geral, podemos constatar que o município de Caxias do Sul obteve escores um pouco melhores do que o município de Novo Hamburgo, sendo mais expressivos os indicadores de "confiança interpessoal" $(0,313)$ e o "conhecimento sobre grupos no bairro" $(0,335)$. Para o contexto de Novo Hamburgo, apenas o indicador "confiança nas instituições" $(0,338)$ apresentou um escore um pouco acima, em relação a Caxias do Sul. O indicador "redes de amizade" ficou idêntico nos dois municípios $(0,250)$.

Constata-se que o indicador "engajamento cívico" foi o mais importante (de maior peso) na determinação do Índice de Capital Social em ambas as cidades. De forma particular, na cidade de Novo Hamburgo, a participação em grupos e associações, pela população, mostra-se bastante inexpressiva $(0,165)$. Neste sentido, dentre as cidades analisadas, a de Caxias do Sul apresentou índice um pouco maior de capital social $(0,366)$ em relação à cidade de Novo Hamburgo $(0,336)$. De uma maneira geral, os valores encontrados apontam para um baixo nível de capital social nas duas regiões, sendo o de Novo Hamburgo mais baixo ainda.

\footnotetext{
${ }^{8}$ O Índice de Capital Social foi construído com a assessoria estatística do Centro de Pesquisa e Planejamento (CPP) da Universidade Feevale.
} 
Em relação à participação no SUAS, no nível mais elementar de participação, o de informação (BORDENAVE, 1983), como podemos observar na Tabela 2, indica que a população do SUAS fala pouco durante uma reunião, pois é o que sugere $60 \%$ da população de Caxias do Sul e $67 \%$ da população de Novo Hamburgo.

Tabela 2 - Participação no SUAS em nível de informação nas cidades de Caxias do Sul e Novo Hamburgo (\%)

\begin{tabular}{|lcc|}
\hline Durante uma reunião, o Sr. costuma: & Caxias do Sul & Novo Hamburgo \\
\hline Falar muito & 17 & 19 \\
Falar pouco & 60 & 67 \\
Não fala & 23 & 24 \\
Total & 100 & 100 \\
\hline
\end{tabular}

Total: Caxias do Sul N = 211 - Novo Hamburgo N = 171 .

Fonte: Próprios autores.

Neste sentido, de uma maneira geral, nos dois municípios, a realidade leva-nos a concordar com Couto et al. (2012), pois, considerando as condições históricas brasileiras de não participação, esse processo é lento e gradual, mas essencial para o avanço da democracia. Entre os desafios que permanecem, constata-se: 1) É esperado que a população apoie as decisões políticas sem questioná-las; 2) É esperado que as propostas apresentadas sejam resolvidas por meio da "adesão", não como espaços de disputas; 3) Ainda persistem lógicas institucionais marcadas pela não participação da população (COUTO et al., 2012).

Em relação à participação em nível de consulta, há uma tendência de que as instituições do SUAS de Caxias do Sul consultem mais a população, pois, considerando os índices de quem se diz sempre convidado e às vezes é convidado, temos um percentual de $31 \%$ da população; e, em Novo Hamburgo, de 23\%, conforme Tabela 3 abaixo.

Tabela 3 - Participação no SUAS em nível de consulta nas cidades de Caxias do Sul e Novo Hamburgo (\%)

\begin{tabular}{|lcc|}
\hline Quando o Sr. é consultado pelo governo local? & Caxias do Sul & Novo Hamburgo \\
\hline Sempre & 7 & 6 \\
Às vezes & 24 & 17 \\
Nunca & 67 & 74 \\
Não sabe & 2 & 3 \\
Total & 100 & 100 \\
\hline
\end{tabular}

Total: Caxias do Sul N = 211 - Novo Hamburgo N = 171 .

Fonte: Próprios autores.

Em se tratando da participação em nível de elaboração, 27\% dos usuários do SUAS de Caxias do Sul foram convidados para reuniões no último ano, enquanto em Novo Hamburgo esse percentual caiu para 23\%, conforme Tabela 4 abaixo. 
Tabela 4 - Participação em nível de elaboração nas cidades de Caxias do Sul e Novo Hamburgo (\%)

\begin{tabular}{|lcc|}
\hline No último ano, o Sr. foi convidado para alguma reunião? & Caxias do Sul & Novo Hamburgo \\
\hline Sim & 27 & 23 \\
Não & 72,5 & 76 \\
Não sabe & 0,5 & 1 \\
Total & 100 & 100 \\
\hline
\end{tabular}

Total: Caxias do Sul N = 211 - Novo Hamburgo N = 171 .

Fonte: Próprios autores.

A tendência de que no contexto de Caxias do Sul a população tenha sido convidada um pouco mais para algum espaço de consulta pode estar relacionada com a recente pesquisa de Couto et al. (2012) sobre os desafios do controle social do SUAS, em nível nacional. Para a autora, em alguns municípios dos Estados do Rio Grande do Sul e do Paraná, têm-se constado iniciativas da gestão pública na criação de novos espaços e estratégias de participação, tais como as pré-conferências e as ouvidorias.

Em relação ao nível máximo de participação, denominado de delegação (BORDENAVE, 1983), observa-se, conforme a Tabela 5 abaixo, que na cidade de Caxias do Sul 26\% da população do SUAS delibera sobre as ações governamentais; em Novo Hamburgo, esse percentual cai um pouco, ficando em $22 \%$.

Tabela 5 - Participação em nível de delegação nas cidades de Caxias do Sul e Novo Hamburgo (\%)

\begin{tabular}{|lcc|}
\hline Quem decide sobre os problemas locais? & Caxias do Sul & Novo Hamburgo \\
\hline Sempre a prefeitura & 25 & 44 \\
Às vezes a prefeitura & 21 & 15 \\
Nem a prefeitura, nem a população & 7 & 8 \\
Muito pouco a população & 4 & 1 \\
As vezes a população & 17 & 10 \\
Sempre a população & 26 & 22 \\
Total & 100 & 100 \\
\hline
\end{tabular}

Total: Caxias do Sul N = 211 - Novo Hamburgo $N=171$.

Fonte: Próprios autores.

Por outro lado, em Novo Hamburgo, os percentuais indicam uma tendência à centralização do governo nas decisões, em que $44 \%$ da população do SUAS avalia que sempre é a prefeitura que decide o que fazer. Em Caxias do Sul, essa relação sugere uma perspectiva bem mais democrática, em que $25 \%$ da população do SUAS indica que sempre é a prefeitura que decide.

Por fim, constata-se que, em relação à participação dos usuários nas conferências, embora esta tenha sido um pouco mais elevada em Novo Hamburgo (23\%) do que em Caxias do Sul (14\%), em ambas as cidades persiste um desafio a ser enfrentado pelos governos e por toda a sociedade, conforme a Tabela 6. 
Tabela 6 - Participação na Conferência Municipal de Assistência Social nas cidades de Caxias do Sul e Novo Hamburgo (\%)

\begin{tabular}{|lcc|}
\hline $\begin{array}{l}\text { No último ano, o Sr. participou da Conferência Municipal } \\
\text { de Assistência Social? }\end{array}$ & Caxias do Sul & Novo Hamburgo \\
\hline Sim & 14 & 23 \\
Não & 74 & 68 \\
Não sabe & 14 & 9 \\
Total & 100 & 100 \\
\hline
\end{tabular}

Total: Caxias do Sul N = 211 - Novo Hamburgo N = 171 .

Fonte: Próprios autores.

A participação dos usuários nas conferências municipais de assistência social passa a ser o espaço privilegiado no SUAS para o exercício democrático nessa política pública. Pereira Flores (2016), ao realizar recente estudo sobre a participação social no SUAS, no Rio Grande do Sul, aponta para o longo período em que o país esteve imerso em regimes e governos patrimonialistas, conservadores e autoritários e para o fato de que ainda apresenta dificuldades de efetivação dos dispositivos previstos na Constituição Cidadã.

Conforme referido anteriormente, o capital social existente em uma sociedade pode ser um elemento de sustentação teórica para o desafio da participação no âmbito das políticas públicas, desta forma: existe associação entre o capital social e participação no SUAS nas cidades em tela? Em concordância com a Tabela 7 abaixo, buscaremos analisar em que medida o acúmulo de capital social existente nos municípios de Caxias do Sul e Novo Hamburgo está associado com a qualidade da participação, nas respectivas cidades. Para tal, vamos utilizar o Teste Qui-Quadrado $\left(X^{2}\right)$, o qual permite testar a significância (grau de associação) entre duas variáveis qualitativas (MEIRELLES, 2012). Desta forma, quando o valor do teste ficar entre 0\% (Sig = 0,00) e 0,5\% (Sig $=0,05)$, deveremos aceitar a hipótese alternativa, qual seja, o capital social está associado com a participação no SUAS, e rejeitar a hipótese nula, qual seja, capital social não está associado com a participação no SUAS.

Tabela 7 - Correlação entre capital social e níveis de participação nas cidades de Caxias do Sul (CS) e Novo Hamburgo (NH) (\%)

\begin{tabular}{|c|c|c|c|c|c|c|}
\hline $\begin{array}{l}\text { Elementos do capital } \\
\text { social } \\
\text { nóve SUAS }\end{array}$ & 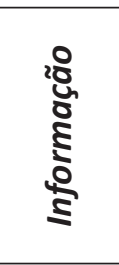 & 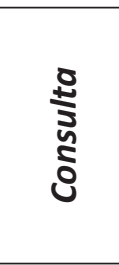 & 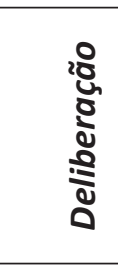 & 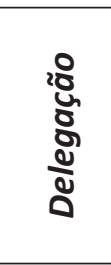 & 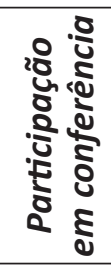 & 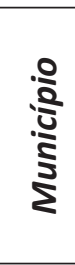 \\
\hline Confiança interpessoal & $\begin{array}{l}0,361 \\
0,504\end{array}$ & $\begin{array}{l}0,734 \\
0,866\end{array}$ & $\begin{array}{l}0,818 \\
0,561\end{array}$ & $\begin{array}{l}0,493 \\
\mathbf{0 , 0 0 4}\end{array}$ & $\begin{array}{l}0,674 \\
0,160\end{array}$ & $\begin{array}{l}\mathrm{CS} \\
\mathrm{NH}\end{array}$ \\
\hline Confiança nos vizinhos & $\begin{array}{l}0,351 \\
\mathbf{0 , 0 3 5}\end{array}$ & $\begin{array}{l}\mathbf{0 , 0 5 0} \\
0,911\end{array}$ & $\begin{array}{l}\mathbf{0 , 0 4 9} \\
0,094\end{array}$ & $\begin{array}{l}\mathbf{0 , 0 3 2} \\
0,091\end{array}$ & $\begin{array}{l}0,825 \\
0,641\end{array}$ & $\begin{array}{l}\mathrm{CS} \\
\mathrm{NH}\end{array}$ \\
\hline Resolver problemas com outras pessoas & $\begin{array}{l}0,013 \\
0,021\end{array}$ & $\begin{array}{l}0,098 \\
0,437\end{array}$ & $\begin{array}{l}\mathbf{0}, 000 \\
0,074\end{array}$ & $\begin{array}{l}0,610 \\
0,139\end{array}$ & $\begin{array}{l}0,760 \\
0,010\end{array}$ & $\begin{array}{l}\mathrm{CS} \\
\mathrm{NH}\end{array}$ \\
\hline Participação em grupos e org. sociais & $\begin{array}{l}0,014 \\
0,000\end{array}$ & $\begin{array}{l}0,561 \\
0,898\end{array}$ & $\begin{array}{l}\mathbf{0 , 0 0 0} \\
0,146\end{array}$ & $\begin{array}{l}\mathbf{0 , 0 4 1} \\
0,057\end{array}$ & $\begin{array}{l}0,076 \\
0,077\end{array}$ & $\begin{array}{l}\mathrm{CS} \\
\mathrm{NH}\end{array}$ \\
\hline Participação em partidos políticos & $\begin{array}{l}0,391 \\
0,211\end{array}$ & $\begin{array}{l}\mathbf{0 , 0 2 1} \\
0,091\end{array}$ & $\begin{array}{l}\mathbf{0 , 0 2 8} \\
0,968\end{array}$ & $\begin{array}{l}0,563 \\
0,197\end{array}$ & $\begin{array}{l}\mathbf{0 , 0 5 0} \\
0,547\end{array}$ & $\begin{array}{l}\mathrm{CS} \\
\mathrm{NH}\end{array}$ \\
\hline
\end{tabular}




\begin{tabular}{|c|c|c|c|c|c|c|}
\hline $\begin{array}{l}\text { Nemeiveis de participação } \\
\text { social } \\
\text { no SUAS }\end{array}$ & 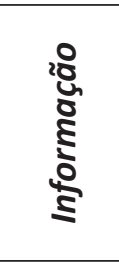 & 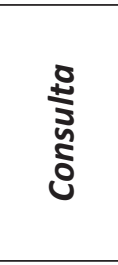 & 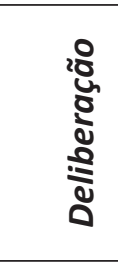 & 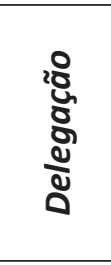 & 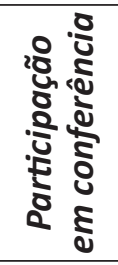 & 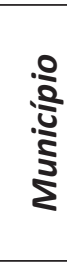 \\
\hline Participação em reunião política & $\begin{array}{l}0,835 \\
0,528\end{array}$ & $\begin{array}{l}\mathbf{0 , 0 2 5} \\
0,116\end{array}$ & $\begin{array}{l}0,203 \\
0,431\end{array}$ & $\begin{array}{l}0,881 \\
0,640\end{array}$ & $\begin{array}{l}\mathbf{0 , 0 3 2} \\
0,074\end{array}$ & $\begin{array}{l}\mathrm{CS} \\
\mathrm{NH}\end{array}$ \\
\hline Participação em comício & $\begin{array}{l}0,437 \\
0,295\end{array}$ & $\begin{array}{l}\mathbf{0 , 0 0 6} \\
0,416\end{array}$ & $\begin{array}{l}\mathbf{0 , 0 0 4} \\
0,326\end{array}$ & $\begin{array}{l}0,978 \\
0,818\end{array}$ & $\begin{array}{l}0,124 \\
0,741\end{array}$ & $\begin{array}{l}\mathrm{CS} \\
\mathrm{NH}\end{array}$ \\
\hline Participação em ass. de moradores & $\begin{array}{l}0,315 \\
\mathbf{0 , 0 0 1}\end{array}$ & $\begin{array}{l}0,098 \\
\mathbf{0 , 0 1 8}\end{array}$ & $\begin{array}{l}0,000 \\
0,021\end{array}$ & $\begin{array}{l}0,037 \\
0,168\end{array}$ & $\begin{array}{l}0,371 \\
0,524\end{array}$ & $\begin{array}{l}\mathrm{CS} \\
\mathrm{NH}\end{array}$ \\
\hline Participação em ONG & $\begin{array}{l}0,319 \\
0,055\end{array}$ & $\begin{array}{l}\mathbf{0 , 0 0 4} \\
0,941\end{array}$ & $\begin{array}{l}0,000 \\
0,029\end{array}$ & $\begin{array}{l}0,175 \\
\mathbf{0 , 0 2 7}\end{array}$ & $\begin{array}{l}0,638 \\
0,214\end{array}$ & $\begin{array}{l}\mathrm{CS} \\
\mathrm{NH}\end{array}$ \\
\hline Participação em orçamento participativo & $\begin{array}{l}0,907 \\
\mathbf{0 , 0 1 4}\end{array}$ & $\begin{array}{l}0,241 \\
0,960\end{array}$ & $\begin{array}{l}\mathbf{0 , 0 2 1} \\
0,430\end{array}$ & $\begin{array}{l}0,133 \\
0,337\end{array}$ & $\begin{array}{l}\mathbf{0 , 0 1 0} \\
0,130\end{array}$ & $\begin{array}{l}\mathrm{CS} \\
\mathrm{NH}\end{array}$ \\
\hline Participação em manifestações ou protestos & $\begin{array}{l}\mathbf{0 , 0 0 5} \\
0,125\end{array}$ & $\begin{array}{l}0,115 \\
0,480\end{array}$ & $\begin{array}{l}0,303 \\
0,987\end{array}$ & $\begin{array}{l}\mathbf{0 , 0 0 4} \\
0,139\end{array}$ & $\begin{array}{l}0,165 \\
\mathbf{0 , 0 2 6}\end{array}$ & $\begin{array}{l}\mathrm{CS} \\
\mathrm{NH}\end{array}$ \\
\hline Participação em greves & $\begin{array}{l}0,172 \\
0,959\end{array}$ & $\begin{array}{l}0,683 \\
0,306\end{array}$ & $\begin{array}{l}0,968 \\
0,369\end{array}$ & $\begin{array}{l}0,112 \\
0,365\end{array}$ & $\begin{array}{l}0,687 \\
\mathbf{0 , 0 0 1}\end{array}$ & $\begin{array}{l}\mathrm{CS} \\
\mathrm{NH}\end{array}$ \\
\hline Participação em ocupação de terreno & $\begin{array}{l}0,268 \\
0,858\end{array}$ & $\begin{array}{l}0,451 \\
0,953\end{array}$ & $\begin{array}{l}\mathbf{0 , 0 2 0} \\
0,998\end{array}$ & $\begin{array}{l}0,603 \\
0,865\end{array}$ & $\begin{array}{l}0,022 \\
8,816\end{array}$ & $\begin{array}{l}\mathrm{CS} \\
\mathrm{NH}\end{array}$ \\
\hline Participação em outros espaços & $\begin{array}{l}0,093 \\
0,573\end{array}$ & $\begin{array}{l}0,266 \\
0,876\end{array}$ & $\begin{array}{l}0,768 \\
0,456\end{array}$ & $\begin{array}{l}0,263 \\
0,844\end{array}$ & $\begin{array}{l}\mathbf{0 , 0 5 0} \\
0,889\end{array}$ & $\begin{array}{l}\mathrm{CS} \\
\mathrm{NH}\end{array}$ \\
\hline
\end{tabular}

Total: Caxias do Sul N = $211-$ Novo Hamburgo N = 171 .

Fonte: Próprios autores.

Constata-se, numa análise comparada entre as cidades, que em Caxias do Sul há um volume superior de associações entre capital social e participação, sobressaindo-se as variáveis confiança nos vizinhos, participação em partidos políticos e participação em grupos e associações comunitárias. Sobre esta última variável, atrelada ao processo de formação sociocultural da cidade de Caxias do Sul, por meio de nossa pesquisa documental realizada, registra-se a existência de 22 associações e clubes, contra 6 em Novo Hamburgo; somado a isto, a cidade de Caxias do Sul conta com 182 associações de moradores, contra 12 da cidade de Novo Hamburgo. Já em Novo Hamburgo a participação no SUAS aparece fortemente associada com a participação em associação de moradores.

Por fim, propomos a análise entre a qualidade do capital social encontrado nas cidades de Caxias do Sul e Novo Hamburgo e a sua relação com a participação no âmbito do SUAS. Para a construção do índice, estabelecemos a mesma metodologia utilizada no Índice de Capital Social. Desta forma, foram estabelecidas algumas afirmações com relação aos níveis de participação nas instituições do SUAS. Para a análise, foram assumidos alguns valores para cada tipo de respostas. Para cada afirmação positiva, foi atribuído valor 2; para cada afirmação negativa, valor 0; e, para cada possibilidade remota ("às vezes", "pouco"), valor 1. O escore geral é dado pela média do percentual de respostas positivas e remotas.

Portanto, a fim de mensurarmos o índice de participação no SUAS, consideramos o seguinte critério: baixo nível de participação no SUAS $-0<I C S \leq 0,5$; médio nível de participação no SUAS $-0,5<I C S \leq 0,8$; alto nível de participação no SUAS - 0,8 < ICS $\leq 1$ (KHAN e SILVA, 2002). Destacamos que a metodologia 
utilizada na elaboração do Índice de Participação no SUAS (IP-SUAS) neste artigo apresenta-se de forma inédita na literatura sobre o SUAS no Brasil, não havendo, assim, outro material publicado que proponha a construção deste índice ${ }^{9}$, conforme a Tabela 8 abaixo.

Tabela 8 - Índice de Participação no SUAS (IP-SUAS) nas cidades de Caxias do Sul e Novo Hamburgo

\begin{tabular}{|lcc|}
\hline \multicolumn{1}{|c}{ Indicador } & Caxias do Sul & Novo Hamburgo \\
\cline { 2 - 3 } & Valor & Valor \\
\hline Participação da população em reunião & 0,386 & 0,380 \\
Quando a população é consultada pelo governo & 0,154 & 0,114 \\
Convite para reunião pelo governo à população & 0,270 & 0,228 \\
Decisão da população nos problemas locais & 0,333 & 0,109 \\
Participação na conferência municipal & 0,137 & 0,234 \\
Total & 0,256 & 0,213 \\
\hline
\end{tabular}

Fonte: Próprios autores.

Dentre as cidades analisadas, Caxias do Sul apresentou um valor um pouco maior do Índice de Participação no SUAS (IP-SUAS), de 0,256, em relação a Novo Hamburgo, com 0,213. Na cidade de Caxias do Sul, em especial, constata-se um melhor desempenho do índice de Participação no SUAS, em virtude de o indicador "decisão da população nos problemas locais" ter tido um melhor escore $(0,333)$ em relação a Novo Hamburgo $(0,109)$. Neste caso, como descentralização e participação passam a ser os principais pilares do reordenamento das políticas públicas, em especial para o SUAS, na cidade de Caxias do Sul a população demonstra ter encontrado melhores condições para participar das decisões do Estado, em nível local.

O percentual alcançado neste indicador também aponta que a realidade do SUAS de Caxias do Sul caminha ao lado de outras experiências de gestão do SUAS no Rio Grande do Sul, em que se constata uma tendência de criação de novos espaços de participação local (COUTO et al., 2012). Neste sentido, Couto et al. (2012) acredita que a organização a partir de espaços locais deverá fortalecer a participação da população nas políticas públicas e a reinvindicação pela melhoria da qualidade de vida, na perspectiva de que a cidade é o espaço de todos. Por outro lado, na cidade de Novo Hamburgo, o indicador "participação na Conferência Municipal de Assistência Social" $(0,234)$ teve melhor desempenho do que na região de Caxias do Sul $(0,137)$, apontando uma tendência maior para a participação em espaços formais propostos pelo governo municipal. Os demais indicadores mantêm-se com percentuais bastante próximos, sendo que o IP-SUAS na cidade de Caxias do Sul revela-se um pouco mais elevado, muito embora em ambas as cidades se constate uma baixa participação no SUAS.

Desta forma, estatisticamente podemos aceitar nossa hipótese alternativa, qual seja, que existe relação positiva entre capital social e a qualidade da participação no SUAS. Numa perspectiva comparada, em Caxias do Sul, constatamos um volume de 26 associações significativas $(\leq 0,05)$; Em Novo Hamburgo, este volume é bem menor, caindo para 13 associações significativas $(\leq 0,05)$. Desta forma, constata-se um estoque bem maior de capital social na cidade de Caxias do Sul e, consequentemente, uma melhor qualidade na participação no SUAS.

${ }^{9} \mathrm{O}$ Índice de Participação no SUAS foi construído com a assessoria estatística do Centro de Pesquisa e Planejamento (CPP) da Universidade Feevale. 


\section{CONSIDERAÇÕES FINAIS}

Além do importante papel das instituições, das leis e dos desafios orçamentários dos governos, o capital social é também uma variável extremamente relevante na análise da participação nas políticas públicas. Esta relevância ocorre já que o capital social capta fatores mais intangíveis, contudo fundamentais, que não podem ser negligenciados nas novas discussões sobre as políticas, em especial no seu aspecto de participação. À luz da teoria do capital social, é possível olharmos a realidade, identificando que os recursos sociais, entre eles, a confiança interpessoal, o envolvimento cívico e a participação, estão presentes nas instituições que se constituíram eficazes e democráticas.

Neste sentido, nosso artigo levou-nos a compreender que os elementos do capital social, entre eles, a confiança nas pessoas, a confiança nos vizinhos, a participação em grupos e organizações e a capacidade de resolução de problemas com outras pessoas, têm relação positiva com a participação no SUAS. Na cidade de Caxias do Sul, a análise dos dados permite-nos sugerir que a participação em associação de moradores, resolução de problemas com outras pessoas, confiança nos vizinhos e engajamento cívico estão diretamente ligados à qualidade da participação no SUAS. Para a cidade de Novo Hamburgo, a participação em associação de moradores aparece diretamente ligada à participação no SUAS. Desta forma, a presença destes recursos sociais, com mais "peso" no contexto da cidade de Caxias do Sul, leva-nos, consequentemente, à constatação de um acúmulo maior de capital social nesta cidade, em relação à cidade de Novo Hamburgo.

Neste sentido, a participação em seu nível de informação, no contexto da cidade de Novo Hamburgo, alcança escores melhores. Contudo, quando observamos os níveis mais sofisticados de participação, como consulta, elaboração e delegação, na cidade de Caxias do Sul, alcança-se melhores resultados. Assim, concordando com Pase (2016), o qual indica que o capital social torna-se uma categoria que pode potencializar os instrumentos de qualificação da democracia, a realidade estudada sugere que, na medida em que cresce o estoque de capital social, aumenta a participação nas políticas públicas, em especial, no SUAS.

\section{REFERÊNCIAS}

ARROW, K. J. The limits of Organization. London: Norton \& Company, 1974.

BANDEIRA, P. S. Algumas Hipóteses sobre as Causas das Diferenças Regionais quanto ao Capital Social no Rio Grande do Sul. In: CORREA, S. M. S. Capital social e desenvolvimento regional. Santa Cruz do Sul: Ed Edunisc, 2003. p. 15-59.

BAQUERO, M. Qual Democracia para a América Latina: capital social e empoderamento são a resposta? Porto Alegre: Ed. UFRGS, 2013.

BAQUERO, M.; GONZALEZ, R. S. Cultura política, mudanças econômicas e democracia inercial: uma análise pós-eleições de 2014. Opin. Publica, v. 22, n. 3, p. 492-523. 2016.

BAQUERO, M.; PRÁ J. A democracia brasileira e a cultura política no Rio Grande do Sul. Porto Alegre: Ed. UFRGS, 2007.

BARBETTA, P. Estatística aplicada às ciências sociais. Florianópolis: Edusfc, 2010. 
BASTOS, F. A. A inserção da odontologia em quatro municípios em gestão plena do Sistema Municipal do SUS no Rio Grande do Sul: um estudo de avaliação institucional. 2008. Tese (Doutorado em Odontologia) - Universidade Luterana do Brasil, Canoas, RS, 2008.

BOBBIO, N.; MATTEUCCI, N.; PASQUINO, G. Dicionário de política. 11. ed. Brasília: UNB, 1998.

BORDENAVE, J. E. D. O que é participação. São Paulo: Brasiliense, 1983.

BOURDIEU, P. Le capital social. Actes de la Recherche en Sciences Sociales, v. 31, p. 2-3, 1980.

BOZEMAN, B.; PANDEY, S. K. Public management decision making: effects of decision content. Public Administration Review, v. 64, n. 5, p. 553-65, 2004.

BRASIL. Ministério do Desenvolvimento Social e Combate a Fome. Secretaria Nacional de Assistência Social. Política Nacional de Assistência Social - PNAS. Brasília, DF, 2014.

BRASIL. Ministério do Desenvolvimento Social e Combate à Fome. Secretaria Nacional de Assistência Social. Norma Operacional Básica NOB - SUAS. Brasília, DF, 2005. Disponível em: https://www.mds.gov. br/webarquivos/arquivo/assistencia_social/nob_suas.pdf. Acesso em: maio 2018.

BRASIL. Presidência da República. Assembleia Legislativa. Constituição da República Federativa do Brasil. Brasília, DF, 5 out. 1988. Disponível em: http://www.planalto.gov.br /ccivil_03/constituicao/ ConstituicaoCompilado.html. Acesso em: abr. 2015.

CARAGNATO, L. Presença e contribuição dos afro-descendentes no município de Caxias do Sul - 1875 a 1950. MÉTIS: história \& cultura, Caxias do Sul, v. 9, n. 17, p. 201-15, jan./jun. 2010.

COLEMAN, J. S. Social capital in the creation of human capital. The American Journal of Sociology, v. 94, Supplement: Organizations and Institutions: Sociological and Economic Approaches to the Analysis of Social Structure, p. 95-120, 1988.

COUTO, B. R; YAZBEK, M. C.; SILVA e SILVA, M. O.; RAICHELIS, R. O Sistema Único de Assistência Social no Brasil: uma realidade em movimento. 3.ed. São Paulo: Cortez, 2012.

DATA FOLHA. Opinião pública: avaliação de governo. São Paulo, 3 jan. 2019. Disponível em: http:// datafolha.folha.uol.com.br/opiniaopublica/avaliacaodegoverno/presidente/jairbolsonaro/indice-1.shtml. Acesso em: 3 jan. 2019.

DOWBOR, L. A reprodução Social - III: descentralização e participação - as novas tendências. Rio de Janeiro: Vozes, 2003.

FERRAZ, F. Brasil: a cultura política de uma democracia mal resolvida. Porto Alegre: Ad2000, 2016.

FUKUYAMA, F. Confiança: as virtudes sociais e a criação da prosperidade. Rio de Janeiro: Ed. Rocco, 1996.

GERTZ, R. Colonização: segunda fase. In: CARELI, S. S.; KNIERIM, L. C. (Org.). Releituras da História do Rio Grande do Sul. Porto Alegre: CORAG. 2011.

GIDDENS, A. (Org). O debate global sobre a terceira via. São Paulo: UNESP, 2007.

GOHN, M. da G. Conselhos gestores e participação sociopolítica. São Paulo: Ed. Cortez, 2011.

GUSTAFSSON, G. Symbolic and pseudo policies as responses to diffusion of Power. Policy sciences, v. 5, n. 3, p. 269-87, 1983. 
HANIFAN, L. J. The rural school community center. In: AMERICAN ACADEMY OF POLITICAL AND SOCIAL SCIENCE, 67., New Possibilities in Education. Anais [...]. p. 130-38, 1916.

HERÉDIA, V. B. M. O mito do imigrante no imaginário da cultura. MÉTIS: história \& cultura, v. 4, n. 8, p. 233-44, jul./dez. 2005

INGLEHART, R. Modernización y posmodernización: el cambio cultural, económico y político en 43 sociedades. Madrid: Centro de Investigaciones Sociológicas/Siglo Veintiuno, 2001.

INSTITUTO BRASILEIRO DE GEOGRAFIA E ESTATíSCA. Caracterização dos municípios do Estado do Rio Grande do Sul. Brasília, DF: IBGE, 2012. Disponível em: https://cidades.ibge.gov.br/brasil/rs/panorama. Acesso em: jan. 2018.

KHAN, A. S.; SILVA, L. M. R. Avaliação do Projeto São José no estado do Ceará: estudo de Caso. Fortaleza: UFC/CCA/DEA, 2012.

LAHERA, E. Introducción a las políticas públicas. Chile: Fondo de Cultura Económica, 2012.

LOWI, T. J. American Business, public policy, case studies, and political theory. World Politics, v. 16, n. 4, p. 667-715, 1964.

MEIRELLES, M. O uso do SPSS (Statistical Package for the Social Sciences) na Ciência Política: uma breve introdução. Revista Pensamento Plural, Pelotas, n. 15, p. 65-91, jan./jun. 2012.

MICELI, S. (Org.). O que ler na ciência social brasileira (1970-1995). São Paulo: Ed. Sumaré/AMPOCS; Brasília: Capes, 1999. v. 1 Antropologia.

MILL, S. O governo representativo. São Paulo: Escala, 2001.

NORTH, D. Institutions, institutional change and economic performance. EUA: Cambridge University Press, 2007.

OLSON, M. A lógica da ação coletiva: os benefícios públicos e uma teoria dos grupos sociais. São Paulo: EDUSP. 1999.

PASE, H. L. Capital social e desenvolvimento: a experiência do Rio Grande do Sul. Pelotas: Ed. UFPEL, 2016.

PASE, H. L.; SANTOS, E. R. Capital social e políticas públicas na América Latina. In: BAQUERO, Marcello (Org.). Cultura(s) política(s) e democracia no século XXI na América Latina. Porto Alegre: UFRGS, 2011. p. 69-93. v. 1.

PEREIRA FLORES, A. P. Participação social: uma realidade nos processos de gestão da Política de Assistência Social em municípios do Rio Grande do Sul? 2016. Dissertação (Mestrado em Serviço Social) - Pontifícia Universidade Católica do Rio Grande do Sul, Porto Alegre, RS, 2016.

PESAVENTO, S. J. História do Rio Grande do Sul. Porto Alegre: Mercado Aberto. 1980.

PUTNAM, R. Comunidade e democracia: a experiência da Itália moderna. São Paulo: FGV, 2000.

RIBEIRO, I; FERNANDES, E; RIBEIRO, H. A importância do capital social para o desenvolvimento de uma região. Revista RURIS, Campinas, v. 6, n. 1, mar. 2012.

ROUSSEAU, J. The social contract. New York: Penguin Books. 1968 
SANTOS, M. de O.; ZANINI, M. C. C. Especificidades da Identidade de descendentes de italianos no sul do Brasil: breve análise das regiões de Caxias do Sul e Santa Maria. Revista Antropolítica, Niterói, n. 27, p. 21-41, 2009. SANTOS, E. Democracia e desenvolvimento: desafios da sociedade gaúcha. ljuí: Edjuí. 2013.

SANTOS E.; NUNES, M. F. Capital Social e políticas públicas: um estudo comparado no Vale do Rio dos Sinos. Revista de Administração Pública, Rio de Janeiro, v. 50, n. 1, p. 129-49, jan./fev. 2016.

SCHÜTZ, M. M. E. Novo Hamburgo: sua história, sua gente. Porto Alegre: Pollott, 1976.

SECCHI, L. Políticas Públicas: conceitos, esquemas de análise, casos práticos. São Paulo: Cengage Learning, 2014.

SOUZA, C. Políticas públicas: uma revisão da literatura. Revista Sociologias, n. 16, p. 20-45, 2006.

STASSEN, J. F. Exclusion and participation: can the excluded become able to participate? Belgium: Université de Liége, 1999.

SZRETER S. Social Capital: critical perspectives. EUA: OXFORD University Press, 1999.

TABELLINI, G. Culture and institutions: economic development in the regions of Europe. EUA: CESIFO, 2005.

TOCQUeVILlE, A. A democracia na América. Tradução, prefácio e notas de Neil Ribeiro da Silva. 2. ed. Belo Horizonte: Itatiaia; São Paulo: Edusp, 1998.

WILSON, J. Q. American government: institutions and policies. Lexington: Heart \& Co., 1983.

WITTMANN, M. L.; RAMOS, M. P. (Org.). Desenvolvimento regional: capital social, redes e planejamento. Santa Cruz do Sul: EDUNISC, 2004.

\section{Sobre os autores:}

Douglas Marques: Pós-doutorando, doutor e mestre em Diversidade Cultural e Inclusão Social pela Universidade Feevale. Especialista no Pensamento Marxista pela Faculdade Porto-Alegrense (FAPA). Graduado em Serviço Social pela Universidade Luterana do Brasil (ULBRA). Colaborador do grupo de pesquisa Metropolização e Desenvolvimento Regional da Universidade Feevale. Professor da Universidade Estadual de Maringá (UEM), no Paraná, e professor visitante do curso de Pós-Graduação em Diversidade e Desenvolvimento de Práticas Inclusivas da Universidade La Salle/RS. E-mail: douglas.sersocial@gmail.com, Orcid: http://orcid.org/0000-0001-9679-7584

Everton Rodrigo Santos: Pós-Doutor, doutor e mestre em Ciência Política pela Universidade Federal do Rio Grande do Sul (UFRGS). Professor titular e pesquisador no Programa de Pós-Graduação em Diversidade Cultural e Inclusão Social da Universidade Feevale. E-mail: chabert89@gmail.com, Orcid: http://orcid.org/0000-0002-6270-3196

Cristina Ennes da Silva: Doutora em História Ibero-Americana pela PUC/RS. Mestrado e Graduação em História pela Universidade do Vale do Rio dos Sinos (UNISINOS). Professora titular na graduação de Teoria da História e História Medieval e do Programa de Pós-Graduação Stricto Sensu Processos e Manifestações Culturais. Pesquisadora do Grupo de Pesquisa em Cultura e Memória da Comunidade da Universidade Feevale. E-mail: crisennes@feevale.br, Orcid: http://orcid.org/0000-0002-3074-7178 